\title{
Síndrome metabólica: prevalência e associação com doenças cardiovasculares
}

\section{Metabolic syndrome: prevalence and association with cardiovascular diseases}

\author{
Mariana Fernandes Costa' \\ Joyce do Valle ${ }^{2}$ \\ ' Nutricionista. Instituto de Nutrição, \\ Universidade do Estado do Rio de Janeiro. \\ 2 Professora Adjunta do Instituto de Nutrição da \\ Universidade do Estado do Rio de Janeiro e da \\ Faculdade de Nutrição da Universidade Federal \\ Fluminense. \\ Correspondência / Correspondence \\ Joyce do Valle \\ E-mail: valle@ueri.br
}

\section{Resumo}

O objetivo deste trabalho foi investigar a prevalência da síndrome metabólica e sua associação com doenças cardiovasculares. Foram avaliados pacientes atendidos no ambulatório de nutrição de um hospital municipal de Niterói, no período de sete meses, sendo somente indivíduos entre 30 e 74 anos sem diagnóstico prévio de cardiopatia. A síndrome metabólica está presente na combinação de pelo menos três ou mais componentes: obesidade abdominal, hipertrigliceridemia, baixo HDL-colesterol, hipertensão arterial e hiperglicemia de jejum. Dados antropométricos e bioquímicos foram coletados. O Escore de Framingham indicou o risco cardiovascular em dez anos. Dos 36 pacientes, 80,56\% eram mulheres. A frequência de síndrome metabólica nas mulheres foi de $89,66 \%$, e nos homens, de 42,86\%. Apresentavam hipertensão arterial sistêmica, 91,7\%; diabetes tipo 2, 77,8\%; e sobrepeso, $86,1 \%$. Obesidade central, hipertrigliceridemia e hiperglicemia estavam mais presentes nas mulheres; hipertensão arterial e baixo HDL-colesterol foram mais frequentes nos homens. As mulheres apresentaram em média 14,97 $\pm 8,39 \%$ de risco cardiovascular, e os homens, $22,00 \pm 17,03 \%$. A correlação entre a síndrome metabólica e o risco cardiovascular foi estatisticamente significativa. A dobra cutânea abdominal está somente associada a variáveis antropométricas. A síndrome metabólica esteve presente em $80,56 \%$ dos indivíduos estudados, estando diretamente relacionada ao alto risco cardiovascular. Os resultados apontam a importância do tratamento preventivo e da redução dos fatores de risco para doenças cardiovasculares, como também o reconhecimento de indivíduos portadores de síndrome metabólica.

Palavras-chave: Síndrome metabólica. Doenças cardiovasculares. Fator de risco. 


\section{Abstract}

This study aimed to investigate the prevalence of metabolic syndrome and the association with cardiovascular disease. The patients evaluated were individuals aged 30-74 years without previous diagnosis of cardiovascular disease, treated in the Nutrition clinic of the municipal hospital in Niterói city for seven months. The metabolic syndrome happens with the combination of three or more conditions such as high blood pressure, high blood glucose, high plasma triglycerides, low HDL cholesterol, and high waist circumference. Anthropometric and biochemical variables were collected. The estimate of cardiovascular risk in ten years was obtained by the Framingham score. Among the 36 patients, $80.56 \%$ were women. The frequency of metabolic syndrome in women was $89.66 \%$ and $42.86 \%$ in men; $91.67 \%$ had hypertension; $77.78 \%$ were diabetic type II; $86.11 \%$ had overweight. High waist circumference, high plasma triglycerides and high blood glucose were more prevalent in women; hypertension and low HDL cholesterol were more prevalent in men. The women had presented in average $14,97 \pm 8.39 \%$ of cardiovascular risk, and men, 22,00 $\pm 17,03 \%$. The correlation between metabolic syndrome and cardiovascular risk was statistically significant. The abdominal skinfold is only associated with anthropometric variables. The metabolic syndrome was present in $80.56 \%$ of the individuals, directly related to high cardiovascular risk. The results indicate the importance of preventive treatment and reduction of risk factors for cardiovascular disease, as well as the recognition of individuals with metabolic syndrome.

Key words: Metabolic syndrome. Cardiovascular disease. Risk factors.

\section{Introdução}

A síndrome metabólica é uma condição em que os fatores de risco para doenças cardiovasculares e diabetes mellitus ocorrem no indivíduo. Obesidade abdominal, hipertensão arterial sistêmica, dislipidemia e distúrbio glicêmico são seus principais componentes, ${ }^{1,2,3}$

As doenças cardiovasculares são as principais causas de morbi-mortalidade nos países desenvolvidos e em desenvolvimento. No Brasil, o perfil da mortalidade proporcional por 
grandes grupos de causas vem se modificando gradualmente desde a década de 1940 até os dias atuais. Observa-se redução progressiva da importância relativa das doenças infecciosas e parasitárias (cerca de 70\%) e aumento concomitante das doenças do aparelho circulatório e das doenças neoplásicas, tanto nos países desenvolvidos como em desenvolvimento. ${ }^{4}$ (OLIVEIRA, 2003).

A Organização Mundial da Saúde ${ }^{5}$ definiu a síndrome metabólica quando o indivíduo possuir dois ou mais componentes: regulação alterada da glicose ou diabetes mellitus e/ ou resistência à insulina; pressão arterial elevada; hipertrigliceridemia; HDL-colesterol baixo; obesidade central e o índice de massa corpórea elevado; microalbuminúria. Essa definição preconiza como ponto de partida a avaliação da resistência à insulina ou do distúrbio do metabolismo da glicose, o que dificulta sua utilização.

O Third Report of the National Cholesterol Education Program Expert Panel on Detection, Evaluation and Treatment of High Blood Cholesterol in Adults (NCEP-ATP III $)^{6}$ publicou uma definição com perspectivas mais clínicas, representada pela combinação de pelo menos três dos componentes: obesidade abdominal, hipertrigliceridemia, baixo HDL-Colesterol, hipertensão arterial e hiperglicemia de jejum. ${ }^{6}$ Por sua simplicidade e praticidade, é a definição recomendada, em 2004, pela I Diretriz Brasileira de Diagnóstico e Tratamento da
Síndrome Metabólica (I-DBSM). ${ }^{7}$ A definição do NCEP-ATP III não exige a comprovação de resistência à ação da insulina, sendo referência para uso clínico. ${ }^{8}$

Este trabalho tem por objetivo investigar a prevalência da síndrome metabólica e sua associação com as doenças cardiovasculares, bem como descrever os componentes fisiopatológicos da síndrome metabólica, estimar o risco cardiovascular e verificar se há associação entre parâmetros antropométricos e outras variáveis analisadas em pacientes adultos acompanhados em ambulatório de nutrição.

\section{Metodologia}

Este estudo é do tipo transversal, no qual foram avaliados 36 indivíduos entre 30 e 74 anos, de ambos os sexos, de todas as classes sociais e cor, independentemente do diagnóstico clínico, atendidos no ambulatório de nutrição de um hospital municipal em Niterói, no período de sete meses. Apenas os pacientes que já apresentavam cardiopatia foram excluídos. Os resultados de exames bioquímicos dos últimos seis meses foram coletados através dos registros nos prontuários. Como critério de diagnóstico da síndrome metabólica, foi adotada a definição elaborada pelo NCEP-ATP III. ${ }^{6}$ Os componentes e os pontos de corte específicos estão demonstrados no quadro 1. 
Quadro 1. Definição de síndrome metabólica segundo NCEP-ATP III

\begin{tabular}{|l|l|}
\hline Três ou mais de: & \\
\hline Circunferência de cintura & \\
\hline Homens & $>102 \mathrm{~cm}$ \\
\hline Mulheres & $>88 \mathrm{~cm}$ \\
\hline Triglicerídeos & $\geq 150 \mathrm{mg} / \mathrm{dL}$ \\
\hline HDL-colesterol & \\
\hline Homens & $<40 \mathrm{mg} / \mathrm{dL}$ \\
\hline Mulheres & $<50 \mathrm{mg} / \mathrm{dL}$ \\
\hline Pressão arterial & $\geq 130 \mathrm{ou} \geq 85 \mathrm{mmHg}$ \\
\hline Glicemia de jejum & $\geq 110 \mathrm{mg} / \mathrm{dL}(6,1 \mathrm{mmol} / \mathrm{L})$ \\
\hline
\end{tabular}

O projeto foi analisado e aprovado pelos Comitês de Ética da Faculdade de Medicina da UFF (n⿳⺈ 197/05-CEP/HUAP) e do Hospital Universitário Pedro Ernesto da UERJ ( $n^{\underline{o}}$ 1.347-CEP/HUPE). Todos os participantes assinaram um termo de consentimento livre e esclarecido, permitindo sua participação na pesquisa e autorizando a divulgação dos resultados obtidos no estudo.

Todos os pacientes foram submetidos à aferição de pressão arterial em duas ocasiões, conforme as Diretrizes Brasileiras de Hipertensão Arterial. ${ }^{9}$ (2004). Vale ressaltar que a presença de diabetes mellitus não excluiu o diagnóstico de síndrome metabólica.

A medida da circunferência de cintura foi realizada com auxílio de uma trena antropométrica na metade da distância entre a crista ilíaca e o rebordo costal inferior. Também foi aferida a dobra cutânea abdominal, com a utilização de um adipômetro da marca Lange ${ }^{\circledR}$, em que a dobra é destacada verticalmente $2 \mathrm{~cm}$ lateral à cicatriz umbilical. ${ }^{10}$

Os valores de massa corporal e estatura foram mensurados por meio da utilização de balança e estadiômetro da marca Filizola ${ }^{\circledR}$, estando o paciente descalço e com o mínimo de roupa possível. O índice de massa corporal (IMC) foi calculado através da fórmula: $\mathrm{IMC}=$ peso/ altura $^{2}\left(\mathrm{Kg} / \mathrm{m}^{2}\right)$, para a classificação do estado nutricional, usando os pontos de corte da OMS. ${ }^{11}$

Para estimativa de risco cardiovascular, foi utilizado o Escore de Risco de Framingham (ERF), que estima em valores percentuais o risco absoluto de eventos coronarianos (morte, infarto agudo do miocárdio e angina de peito) em dez anos, além de ser validado e sugerido como método de estimação de risco pelas Diretrizes Brasileiras sobre Dislipidemias e Prevenção da Aterosclerose. ${ }^{12-14}$ A classificação e pontuação destas variáveis foram feitas de acordo com o quadro 2. 
Quadro 2. Estratificação de risco cardiovascular pelo Escore de Framingham

\begin{tabular}{|ccc|}
\hline \multicolumn{3}{|c|}{ Passo 1 } \\
\hline Idade & Homens & Mulheres \\
\hline $30-34$ & -1 & -9 \\
\hline $35-39$ & 0 & 4 \\
\hline $40-44$ & 1 & 0 \\
\hline $45-49$ & 2 & 3 \\
\hline $50-54$ & 3 & 6 \\
\hline $55-59$ & 4 & 7 \\
\hline $60-64$ & 5 & 8 \\
\hline $65-69$ & 6 & 8 \\
\hline $70-74$ & 7 & 8 \\
\hline
\end{tabular}

\begin{tabular}{|ccc|}
\hline & Passo 5 & \\
\hline Diabetes & Homens & Mulheres \\
\hline Sim & 2 & 4 \\
\hline Não & 0 & 0 \\
\hline
\end{tabular}

\begin{tabular}{|ccc|}
\hline \multicolumn{3}{|c|}{ Passo 2 } \\
\hline $\begin{array}{c}\text { Colesterol } \\
\text { Total }(\mathbf{m g} / \mathbf{d L})\end{array}$ & Homens & Mulheres \\
\hline$<160$ & -3 & -2 \\
\hline $160-199$ & 0 & 0 \\
\hline $200-239$ & 1 & 1 \\
\hline $240-279$ & 2 & 1 \\
\hline 2280 & 3 & 3 \\
\hline
\end{tabular}

\begin{tabular}{|ccc|}
\hline \multicolumn{3}{|c|}{ Passo 6 } \\
\hline Fumo & Homens & Mulheres \\
\hline Sim & 2 & 4 \\
\hline Năo & 0 & 0 \\
\hline
\end{tabular}

\begin{tabular}{|c|}
\hline Passo 7 \\
\hline $\begin{array}{c}\text { Idade + CT + HDL }-\mathrm{C}+\text { PAS ou PAD + DM + Fumo } \\
\text { total de pontos }\end{array}$ \\
\hline
\end{tabular}

\begin{tabular}{|c|c|c|}
\hline \multicolumn{3}{|c|}{ Passo 3} \\
\hline HDL-C (mg/dL) & Homens & Mulheres \\
\hline$<35$ & 2 & 2 \\
\hline $35-41$ & 1 & 2 \\
\hline $45-49$ & 0 & 1 \\
\hline $50-59$ & 0 & 0 \\
\hline 260 & -1 & -3 \\
\hline
\end{tabular}

\begin{tabular}{|cccc|}
\hline \multicolumn{4}{|c|}{ Passo $4^{*}$} \\
\hline $\begin{array}{c}\text { PAS } \\
(\mathrm{mmHg})\end{array}$ & $\begin{array}{c}\text { PAD } \\
(\mathrm{mmHg})\end{array}$ & Homens & Mulheres \\
\hline$<120$ & $<80$ & 0 & -3 \\
\hline $120-129$ & $80-84$ & 0 & 0 \\
\hline $130-139$ & $85-89$ & 1 & 0 \\
\hline $140-159$ & $90-99$ & 2 & 2 \\
\hline 2160 & 2100 & 3 & 3 \\
\hline
\end{tabular}

*Quando osvalores de PAS e PAD discordarem, usar o mais alto.

\begin{tabular}{|c|c|c|c|}
\hline \multicolumn{4}{|c|}{ Passo 8} \\
\hline $\begin{array}{c}\text { Homens } \\
\text { Pontos }\end{array}$ & $\begin{array}{c}\text { Homens } \\
\text { Risco de } \\
\text { DAC em } \\
10 \text { anos } \\
(\%)\end{array}$ & $\begin{array}{c}\text { Mulheres } \\
\text { Pontos }\end{array}$ & $\begin{array}{l}\text { Mulheres } \\
\text { Risco de } \\
\text { DAC em } 10 \\
\text { anos }(\%)\end{array}$ \\
\hline$<-1$ & 2 & $s-2$ & 1 \\
\hline 0 & 3 & -1 & 2 \\
\hline 1 & 3 & 0 & 2 \\
\hline 2 & 4 & 1 & 2 \\
\hline 3 & 5 & 2 & 3 \\
\hline 4 & 7 & 3 & 3 \\
\hline 5 & 8 & 4 & 4 \\
\hline 6 & 10 & 5 & 4 \\
\hline 7 & 13 & 6 & 5 \\
\hline 8 & 16 & 7 & 6 \\
\hline 9 & 20 & 8 & 7 \\
\hline 10 & 25 & 9 & 8 \\
\hline 11 & 31 & 10 & 10 \\
\hline 12 & 37 & 11 & 11 \\
\hline 13 & 45 & 12 & 13 \\
\hline$\geq 14$ & 53 & 13 & 15 \\
\hline & & 14 & 18 \\
\hline & & 15 & 20 \\
\hline & & 16 & 24 \\
\hline & & 17 & 227 \\
\hline
\end{tabular}

Valores estimados para o risco de morte ou infarto do miocárdio em 10 anos (NCEP ATP III 2001).

Fonte: Adaptado da I-DBM,2004.

Para análise estatística dos dados deste estudo, foram aplicados testes estatísticos e fixado um valor de significância de até $5 \%$ de probabilidade $(\mathrm{p}<0,05)$, segundo Rodrigues. ${ }^{15}$ O tratamento estatístico foi realizado através dos softwares GraphPad Instat ${ }^{\circledR}$ e do Excel $2003^{\circledR}$. 


\section{Resultados e discussão}

As tabelas 1 e 2 apresentam, por sexo, as médias aritméticas, desvios padrão, medianas, valor mínimo e valor máximo das variáveis: idade, peso corporal, estatura, Índice de Massa Corporal (IMC), circunferência abdominal, dobra cutânea abdominal, glicose, triglicerídeos, colesterol total, HDL-colesterol, pressão arterial sistólica e pressão arterial diastólica.

Tabela 1. Medida de posição e dispersão das variáveis contínuas em estudo da amostra de mulheres acompanhadas no ambulatório de nutrição de um hospital municipal em Niterói

\begin{tabular}{lccccc}
\hline \multicolumn{1}{c}{ Variáveis } & Média & Mediana & $\begin{array}{c}\text { Desvio- } \\
\text { padrão }\end{array}$ & $\begin{array}{c}\text { Valor } \\
\text { mínimo }\end{array}$ & $\begin{array}{c}\text { Valor } \\
\text { máximo }\end{array}$ \\
\hline Idade (anos) & 56,79 & 58,00 & 11,23 & 32,00 & 74,00 \\
Peso (kg) & 72,97 & 68,00 & 15,65 & 51,90 & 108,00 \\
Estatura (m) & 1,54 & 1,54 & 0,07 & 1,43 & 1,68 \\
IMC (kg/m²) & 30,61 & 30,40 & 5,21 & 22,80 & 44,00 \\
CC (cm) & 100,71 & 101,00 & 13,40 & 62,40 & 125,00 \\
DCA (mm) & 47,88 & 45,30 & 13,01 & 24,50 & 71,40 \\
Glicose de jejum (mg/dL) & 146,55 & 126,00 & 66,10 & 73,00 & 354,00 \\
Triglicerídeos (mg/dL) & 206,93 & 202,00 & 87,62 & 42,00 & 449,00 \\
Colesterol total (mg/dL) & 224,00 & 218,00 & 42,84 & 164,00 & 369,00 \\
HDL-colesterol (mg/dL) & 42,48 & 41,00 & 11,61 & 27,00 & 81,00 \\
PAS (mmHg) & 153,07 & 150,00 & 29,88 & 114,00 & 235,00 \\
PAD (mmHg) & 88,34 & 86,00 & 11,00 & 71,00 & 120,00 \\
\hline
\end{tabular}


Tabela 2. Medida de posição e dispersão das variáveis contínuas em estudo da amostra de homens acompanhados no ambulatório de nutrição de um hospital municipal em Niterói

\begin{tabular}{lccccc}
\hline \multicolumn{1}{c}{ Variáveis } & Média & Mediana & $\begin{array}{c}\text { Desvio } \\
\text { padrão }\end{array}$ & $\begin{array}{c}\text { Valor } \\
\text { mínimo }\end{array}$ & $\begin{array}{c}\text { Valor } \\
\text { máximo }\end{array}$ \\
\hline Idade (anos) & 62,70 & 63,00 & 8,64 & 48,00 & 76,00 \\
Peso (kg) & 78,71 & 78,60 & 12,39 & 62,00 & 97,30 \\
Estatura (m) & 1,70 & 1,70 & 0,07 & 1,58 & 1,77 \\
IMC (kg/m²) & 27,17 & 26,00 & 2,73 & 24,70 & 32,50 \\
CC (cm) & 100,34 & 96,20 & 9,45 & 89,00 & 112,00 \\
DCA (mm) & 32,30 & 30,50 & 10,11 & 20,50 & 44,70 \\
Glicose de jejum (mg/dL) & 135,14 & 103,00 & 67,20 & 80,00 & 247,00 \\
Triglicerídeos (mg/dL) & 114,71 & 90,00 & 93,08 & 43,00 & 311,00 \\
Colesterol (mg/dL) & 160,86 & 166,00 & 31,66 & 113,00 & 215,00 \\
HDL-colesterol (mg/dL) & 41,71 & 40,00 & 10,83 & 29,00 & 61,00 \\
PAS (mmHg) & 160,00 & 144,00 & 32,92 & 131,00 & 223,00 \\
PAD (mmHg) & 91,71 & 84,00 & 19,21 & 75,00 & 132,00 \\
\hline CC = Circunferência de Cintura; DCA $=$ Dobra Cutânea Abdominal; PAS = Pressão Arterial Sistólica; PAD = \\
Pressão Arterial Diastólica.
\end{tabular}

Dentre os 36 indivíduos que participaram do estudo, 80,56\% eram mulheres e 19,44\% homens. Considerando os critérios que caracterizam a síndrome metabólica, segundo o NCEP-ATPIII, constatou-se que $80,56 \%$ apresentavam esta síndrome. A frequência de síndrome metabólica nas mulheres estudadas foi de $89,66 \%$ e nos homens de
42,86\%. Dos componentes da síndrome metabólica, a obesidade central foi mais frequente nas mulheres, mas acrescentamse a este componente a hipertrigliceridemia e a hiperglicemia; nos homens, além da hipertensão arterial, destaca-se o baixo HDLcolesterol. Na figura 1, é possível verificar a distribuição dos componentes da síndrome metabólica. 


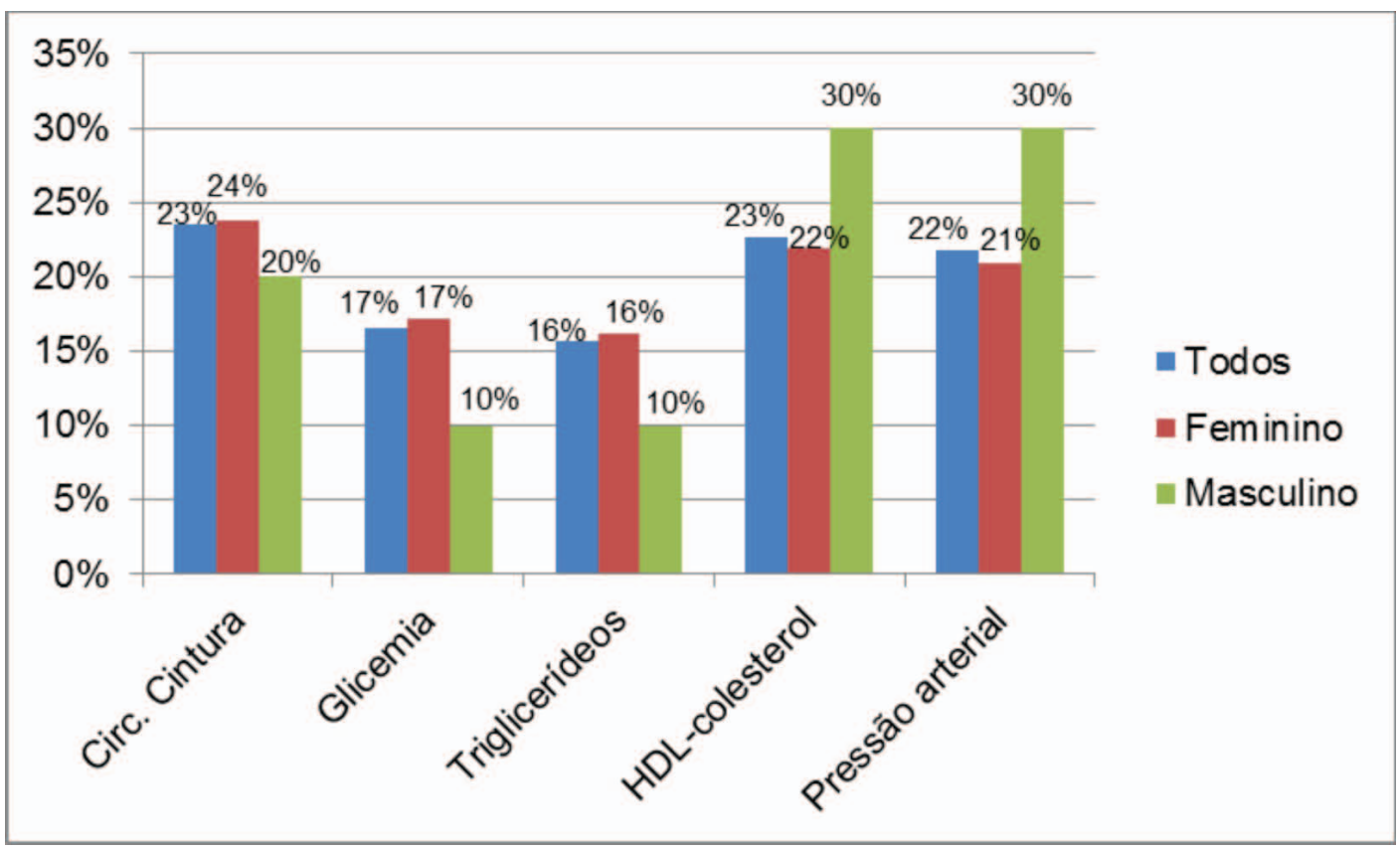

Figura 1. Prevalência dos componentes da síndrome metabólica na amostra de pacientes acompanhados no ambulatório de nutrição de um hospital municipal em Niterói

Verificou-se também que, de todo o grupo estudado, 91,67\% possuíam hipertensão arterial sistêmica e $77,78 \%$ eram diabéticos tipo II. No sexo feminino, 89,66\% eram portadores de hipertensão arterial e 75,86\% apresentavam diabetes mellitus tipo II. Já no sexo masculino, $100 \%$ hipertensos e $85,71 \%$, diabéticos.
De acordo com o IMC, 86,11\% da comunidade apresentaram sobrepeso e 13,89\% possuíam estado nutricional adequado. Dentre a classificação de sobrepeso, 38,89\% estavam na pré-obesidade, $30,56 \%$ em obesidade grau 1, $11,11 \%$ em obesidade grau 2 e $5,55 \%$ em obesidade grau 3 (obesidade mórbida). A figura 2 apresenta a classificação do estado nutricional por sexo. 


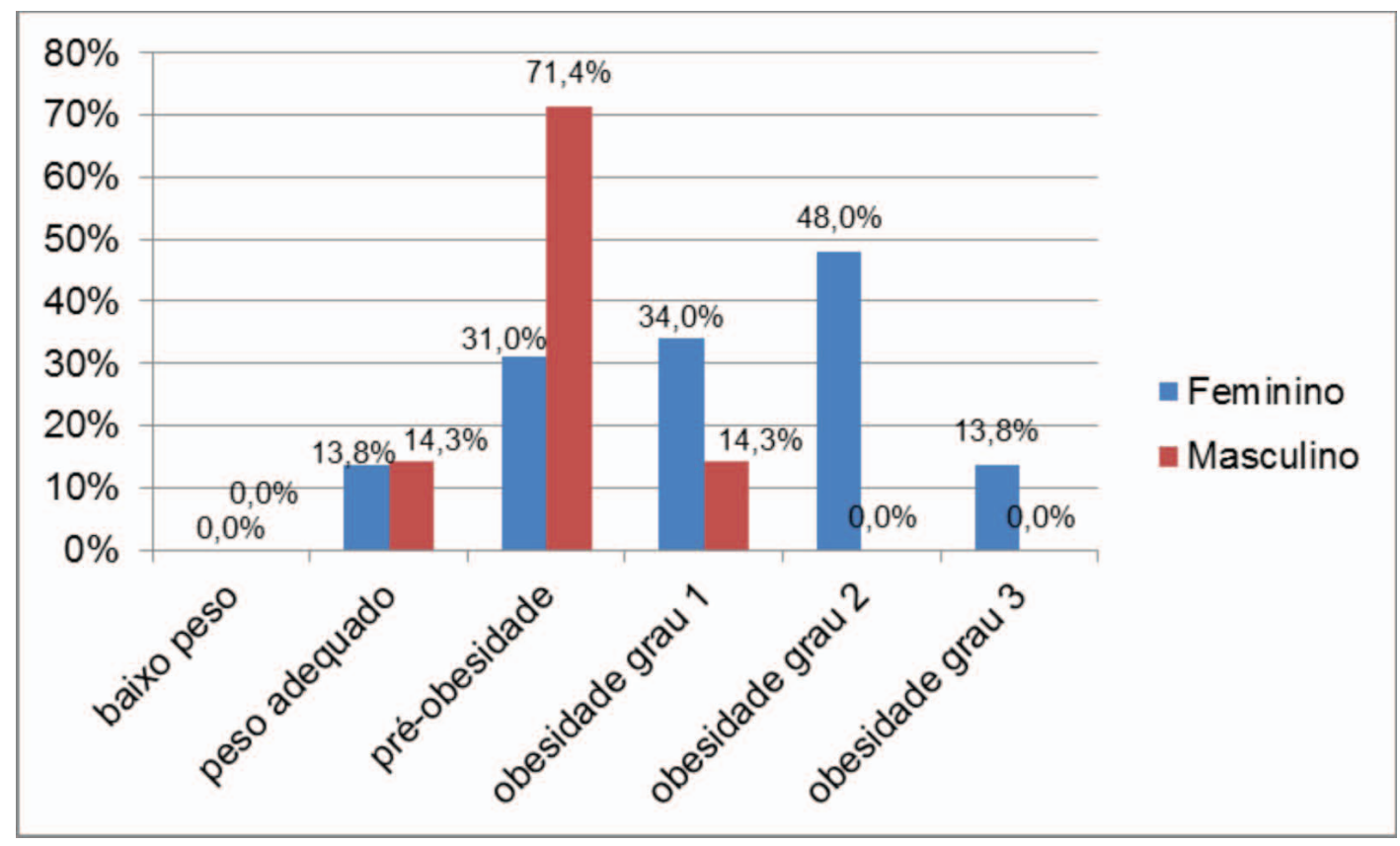

Figura 2. Classificação do estado nutricional por sexo, segundo IMC, na amostra de pacientes acompanhados no ambulatório de nutrição de um hospital municipal em Niterói

As mulheres apresentaram em média $14,97 \pm 8,39 \%$ de risco de desenvolver doença arterial coronariana em dez anos. Já os homens, 22,00 $\pm 17,03 \%$ de risco. O resultado da correlação entre o número de componentes que caracterizam a síndrome metabólica e o percentual de risco cardiovascular, nas mulheres, foi positiva e fraca $(r=0,3691)$, porém estatisticamente significativa $(\mathrm{p}=$ 0,0488). Nos homens, essa correlação foi positiva e forte $(r=0,8825)$, sendo muito significativa $(\mathrm{p}=0,0085)$.

Segundo Ford et al., ${ }^{16}$ estudos considerando a origem étnica dos indivíduos verificaram que mulheres americanas de origem africana e mexicana apresentam maior prevalência de síndrome metabólica do que os homens dos mesmos grupos étnicos. Um estudo brasileiro, produzido por Rocha et al., ${ }^{17}$ com indígenas de duas cidades no Rio Grande do Sul de 
idade maior do que 40 anos destaca que a prevalência da síndrome metabólica foi de $65,3 \%$, sendo mais prevalente no sexo feminino $(\mathrm{p}<0,001)$. Alterações na circunferência abdominal, glicemia de jejum e HDL-colesterol e presença de hipertensão arterial sistêmica, hipertrigliceridemia e obesidade foram associados à síndrome metabólica.

Estudos como o The United Kingdom Prospective Diabetes Study ${ }^{18}$ e The Scandinavian Simvastatin Survival Study ${ }^{19}$ revelaram claramente que alguns fatores, excluindo o controle glicêmico, possuem papel vital nas doenças macrovasculares. Assim, os fatores de risco vasculares são de extrema importância para os diabéticos. Como a maior parte do grupo analisado é constituída de mulheres diabéticas, é importante lembrar que já está muito difundido no meio científico o fato de as mulheres diabéticas apresentarem redução no seu fator de proteção ligado ao sexo para doenças vasculares, embora mecanismos precisos ainda permanecerem incertos. Ademais, foi relatado na literatura, por Sowers ${ }^{20}$ e Howard et al.,, ${ }^{21}$ que as mulheres apresentam mais fatores de risco, o que resulta na manifestação de eventos macrovasculares mais comumente como problemas clínicos.

Vega $^{22}$ citou que métodos diretos para quantificar gordura corporal são melhores do que aqueles que usam o índice de massa corporal como uma medida alternativa para mensurar massa gorda. Nossa análise identificou que, para ambos os sexos, há uma associação direta estatisticamente significativa, entre as variáveis dobra cutânea abdominal e peso; índice de massa corporal; e circunferência de cintura (tabela 3). Entretanto, não se estabeleceu associação estatisticamente significativa entre a dobra cutânea abdominal e dados bioquímicos (glicemia de jejum, trigliceridemia, colesterol total e HDL-colesterol), assim como também não se verificou relação com os valores de pressão arterial sistólica e diastólica e idade.

Além disso, a variável dobra cutânea abdominal também não se mostrou associada ao número de componentes que caracterizam a síndrome metabólica, segundo o NCEP-ATP III, ${ }^{6}$ nem ao percentual de risco de desenvolvimento de doença arterial coronariana em dez anos, estimado pelo escore de risco de Framingham. Desta forma, constatou-se que apenas houve relação direta entre a dobra cutânea abdominal e as demais variáveis antropométricas no grupo estudado.

Lerarioa et al. ${ }^{23}$ estudaram 530 nipobrasileiros (40-79 anos), de primeira e segunda gerações, avaliando medidas antropométricas, pressão arterial, perfil lipídico e teste oral de tolerância à glicose. Ao compararem essas variáveis com ou sem adiposidade central, estas foram compatível com a hipótese de que a deposição abdominal de gordura representa fator de risco para doenças interligadas pela resistência à insulina, inclusive em população de origem oriental. 
Tabela 3. Correlação entre dobra cutânea abdominal e outras variáveis da amostra de pacientes acompanhados no ambulatório de nutrição de um hospital municipal em Niterói.

\begin{tabular}{lcccc}
\hline \multicolumn{1}{c}{ Variáveis $\backslash$ Gênero } & \multicolumn{2}{c}{ Feminino } & \multicolumn{2}{c}{ Masculino } \\
\hline Idade & $\mathrm{r}$ & $\mathrm{p}$ & $\mathrm{r}$ & $\mathrm{p}$ \\
Peso & $-0,2716$ & 0,1541 & 0,2182 & 0,6383 \\
Índice de massa corporal & 0,6289 & $0,0003^{*}$ & 0,8318 & $0,0203^{*}$ \\
Circunferência de cintura & 0,6568 & $0,0001^{*}$ & 0,8369 & $0,0189^{*}$ \\
Glicemia de jejum & 0,5242 & $0,0035^{*}$ & 0,9617 & $0,0005^{*}$ \\
Triglicerídeos & $-0,2500$ & 0,1908 & $-0,4334$ & 0,2950 \\
Colesterol total & 0,1320 & 0,01742 & 0,4048 & 0,3676 \\
HDL-colesterol & $-0,2710$ & 0,1551 & $-0,5439$ & 0,2069 \\
Pressão arterial sistólica & $-0,04989$ & 0,7972 & $-0,6028$ & 0,1519 \\
Pressão arterial diastólica & $-0,06403$ & 0,7414 & 0,3018 & 0,5107 \\
Número de componentes da & 0,2926 & 0,1235 & 0,2849 & 0,5357 \\
síndrome metabólica & 0,1176 & 0,5434 & 0,3919 & 0,3845 \\
Percentual de risco de doença & $-0,1482$ & 0,4431 & 0,1110 & 0,8126 \\
arterial coronariana & & & & \\
*p $<0,05$ & & & & \\
\hline
\end{tabular}

Entretanto, Reaven ${ }^{24}$ pontuou que a obesidade abdominal não é uma manifestação de resistência à insulina, e sim uma variável antropométrica que pode acentuar o grau de resistência à insulina devido ao efeito ainda não direcionado da obesidade generalizada. Esse autor concluiu com a assertiva de que nem todos os sujeitos com sobrepeso são insulino-resistentes, e nem todos os indivíduos insulino-resistentes estão com sobrepeso. Contudo, na população de Framingham, a presença apenas da síndrome metabólica, classificada de acordo com o IIII NCEP, ${ }^{25}$ foi capaz de predizer cerca de $25 \%$ dos novos casos de doença cardiovascular observados em oito anos de seguimento. Segundo Grundy, ${ }^{26}$ ao aplicar a tabela de Framingham à síndrome metabólica, na ausência de diabetes mellitus, o risco para doença coronariana variou de 10 a $20 \%$ em dez anos.

Por outro lado, Wilson ${ }^{27}$ comentou que os investigadores de Framingham, utilizando métodos estatísticos específicos, 
não encontraram diferença significativa da estimativa do risco cardiovascular quando agregaram, ao algoritmo de Framingham, valores da síndrome metabólica, considerados no todo ou em separado, tanto em pacientes diabéticos como em não-diabéticos. Portanto, não há aumento do risco cardiovascular pela adição dos fatores de risco da síndrome metabólica, não contemplados na tabela ao cálculo do escore de Framingham.

Grundy $^{26}$ ainda destacou que é provável que a maior parte do risco associado à síndrome metabólica seja marcado pelos fatores já incluídos na tabela de Framingham. Além desses, a obesidade, os triglicerídeos e a glicose elevada, excluído o diabetes mellitus, não teriam poder de aumentar significativamente o risco cardiovascular. No entanto, quando foi analisado o risco para início recente de diabetes mellitus na população de Framingham, em ambos os sexos, a síndrome metabólica, classificada de acordo com o III NCEP, ${ }^{25}$ foi capaz de explicar quase a metade do risco para diabetes mellitus atribuído à população.

$\mathrm{Na}$ presente pesquisa, foi possível identificar uma associação estatisticamente significativa entre o número de componentes que caracterizam um indivíduo com síndrome metabólica e o percentual de risco do mesmo desenvolver doença arterial coronariana em dez anos. Deste modo, observou-se que, tanto nas mulheres como nos homens, a correlação entre a síndrome metabólica e o percentual de risco cardiovascular calculado através do Escore de Risco de Framinghan é positiva. Isso indica que a definição do NCEP-ATP III de síndrome metabólica é capaz de identificar sujeitos com risco de morbidade e mortalidade cardiovascular aumentados, além de oferecer uma ferramenta que pode ser utilizada para comparação entre resultados de diferentes estudos.

De acordo com Isomaa et al. ${ }^{28}$ e Yip et al., ${ }^{29}$ a síndrome metabólica está associada com o aumento do risco de doenças cardiovasculares e representa um importante critério para identificar indivíduos que devem ser alvo na redução do risco dessas doenças. Reaven ${ }^{24}$ acrescentou que tanto a redução do risco de doença cardiovascular quanto a prevenção do diabetes mellitus poderiam e deveriam ser considerados para todos os pacientes com síndrome metabólica. Por causa do grande número de indivíduos afetados, esses esforços sem dúvida alguma teriam impacto significativo na saúde pública.

\section{Conclusão}

Como principais componentes fisiopatológicos da síndrome metabólica, encontramos: obesidade, resistência insulínica e/ou diabetes mellitus tipo 2, dislipidemia e hipertensão arterial.

A maior parte dos pacientes acompanhados possui síndrome metabólica e alto risco cardiovascular. Nas mulheres, obesidade central, hipertrigliceridemia e hiperglicemia foram mais frequentes. Nos homens, hipertensão arterial e baixo HDL-colesterol tiveram destaque. 
Além disso, foi possível constatar que há uma associação direta entre síndrome metabólica e estimativa para o risco de morte ou infarto do miocárdio em dez anos. Já a dobra cutânea abdominal foi somente associada ao peso, índice de massa corporal e circunferência de cintura, não apresentando relação com as demais variáveis investigadas.

\section{Referências}

1. Goodman E, Daniels SR, Dolan LM. Definition of metabolic syndrome. J Pediatr. 2007; 150(4): 36.

2. Demacker PN. The metabolic syndrome: definition, pathogenesis and therapy. Eur J Clin Invest. 2007; 37(2): 85-9.

3. Lopes HF. Hipertensão arterial e síndrome metabólica: além da associação. Rev Soc Cardiol Estado de São Paulo 2003; 13(1): 64-77.

4. Oliveira GMM et al. Abordagem da Doença Coronariana: aspectos epidemiológicos, diagnósticos e terapêuticos. Rio de Janeiro: Ed. Edson Abdala Saad; 2003. 132 p.

5. Organização Mundial de Saúde. WHO consultation: Definition, diagnosis and classification of diabetes mellitus and its complications. WHO/NCD/NCS/99.2; 31-33.

6. Expert Panel on Detection, Evaluation and Treatment of High Blood Cholesterol in Adults. Executive summary of the Third Report of the National Cholesterol Education Program (NCEP) Expert Panel on Detection, Evaluation and Treatment of High Cholesterol. JAMA. 2001; 285:2486-97.
Mesmo assim, ressalta-se a contribuição deste estudo para os profissionais da área da saúde e pacientes, no sentido de demonstrar a importância do tratamento preventivo e da redução dos fatores de risco para doenças cardiovasculares, como também o reconhecimento de indivíduos portadores de síndrome metabólica.

7. I Diretriz Brasileira de Diagnóstico e Tratamento da Síndrome Metabólica (IDBSM). Rev Bras Hipertens 2005; 7(4):130-1; 149-150.

8. Rodrigues TC, Canani LH, Gross JL. Síndrome Metabólica, Resistência à Ação da Insulina e Doença Cardiovascular no Diabete Melito Tipo 1. Arq Bras Cardiol. 2010; 94(1): 134-39.

9. IV Diretrizes Brasileiras de Hipertensão Arterial. Arq Bras Cardiol. 2003; 82 supl. 4: 1206-52.

10. Heyward VH, Stolarczyk LM. Avaliação da Composição Corporal Aplicada. São Paulo: Manole; 2000. p. 42.

11. Organização Mundial de Saúde. Obesity: preventing and managing the global epidemic. Report of a WHO, Consultation on Obesity. Geneve: WHO; 1998.

12. III Diretrizes Brasileiras sobre Dislipidemia e Diretriz de Prevenção da Aterosclerose do Departamento de Aterosclerose da Sociedade Brasileira de Cardiologia. Arq Bras Cardiol. 2001 Nov; 77 supl. III.

13. Mesquita ET. Paciente vulnerável: epidemias cardiovasculares emergentes. Rio de Janeiro: Lápis de Cor; 2003. 122 p. 
14. Sposito AC et al. IV Diretriz Brasileira sobre Dislipidemias e Prevenção da Aterosclerose: Departamento de Aterosclerose da Sociedade Brasileira de Cardiologia. Arq Bras Cardiol 2007; 88 suppl.1: 2-19.

15. Rodrigues PC. Bioestatística. 3 ed. Rio de Janeiro: EdUFF; 2002. 349 p.

16. Ford ES et al. Prevalence of the metabolic syndrome among US adults. Findings from the Third National Health and Nutrition Examination Survey. JAMA. 2002; 287: 356-59.

17. Rocha AKS et al. Prevalência da síndrome metabólica em indígenas com mais de 40 anos no Rio Grande do Sul, Brasil. Rev Panam Salud Publica 2011; 29(1): 41-5.

18. UK Prospective Diabetes Study Group. Tight blood pressure control and risk of macrovascular and microvascular complications in type 2 diabetes: UKPDS 38. BMJ. 1998; 17:703-13.

19. Girman CJ et al. The metabolic syndrome and risk of major coronary events in the Scandinavian Simvastatin Survival Study (4S) and the Air Force/Texas Coronary Atherosclerosis Prevention Study (AFCAPS/ TexCAPS). Am J Cardiol. 2004; 93: 136-41.

20. Sowers JR. Diabetes mellitus and cardiovascular disease in women. Arch Intern Med. 1998; 158:617-21.

21. Howard BV et al. Adverse effects of diabetes on multiple cardiovascular disease risk factors in women. Diabetes Care 1998; 21: 1258-1265.
22. Vega GL. Obesity, the metabolic syndrome, and cardiovascular disease. Am Heart J. 2002; 142(16): 1108-16.

23. Lerarioa DDG. Excesso de peso e gordura abdominal para a síndrome metabólica em nipo-brasileiros. Rev. Saúde Pública 2002; 36(1): 4-11.

24. Reaven G. Metabolic syndrome. Pathophysiology and implications for management of cardiovascular disease. Circulation 2002; 106: 286-88.

25. Third Report of the National Cholesterol Education Program (NCEP) Expert Panel on Detection, Evaluation, and Treatment of High Blood Cholesterol in Adults (Adult Treatment Panel III or ATP III) final report. Circulation 2002; 106: 3143-421.

26. Grundy SM et al. Definition of the metabolic syndrome. Report of the National Heart, Lung and Blood Institute/American Heart Association Conference on Scientific Issues Related to Definition. Circulation 2004; 109:433-38.

27. Wilson PWF et al. Prediction of coronary heart disease using risk factors categories. Circulation 1998; 97:1837-47.

28. Isomaa B et al. Cardiovascular morbidity and mortality associated with the metabolic syndrome. Diabetes Care 2001; 24: 683-89.

29. Yip P, Facchini FS, Reaven GM. Resistance to insulin mediated glucose disposal as a predictor of cardiovascular disease. J Clin Endocrinol Metab. 1998; 83: 2773-76. 